\title{
PATHOGENICITY AND SEQUENCE ANALYSIS STUDIES SUGGEST POTENTIAL ROLE OF GENE 3 IN VIRULENCE OF SWINE ENTERIC AND RESPIRATORY CORONAVIRUSES
}

\author{
Prem S. Paul, Eric M. Vaughn, and Patrick G. Halbur \\ Iowa State University College of Veterinary Medicine \\ Ames, Iowa 50011
}

\section{SUMMARY}

Coronaviruses have been commonly associated with enteric and respiratory diseases. Two of the swine coronaviruses, namely transmissible gastroenteritis virus (TGEV) and porcine respiratory coronavirus (PRCV), have been extensively studied. TGEV replicates in both the enteric and respiratory tracts and causes enteric disease, whereas, PRCV replicates in the respiratory tract with limited to no replication in the enteric tract. We have isolated PRCV from swine herds with respiratory disease and have reproduced moderate pneumonia in gnotobiotic and conventionally reared pigs with two of the PRCV isolates. We have also identified two PRCV isolates with low virulence. One consistent difference that we have observed between PRCV isolates of different pathogenicities is in gene 3 . The gene 3 is intact in the two virulent PRCV isolates, whereas gene 3 is altered in the two low virulence isolates. A similar observation has been reported for TGEV as a nonpathogenic TGEV mutant with a small plaque morphology had a deletion in gene 3 . We have also observed that one of the low virulence PRCV isolates, IA1894, which has a deletion in gene 3, replicates poorly in cell cultures. Collectively these studies suggest that gene 3 may be an important determinant for in vivo virulence and in vitro replication of coronaviruses.

\section{INTRODUCTION}

Coronaviruses are a common cause of disease in many species of animals. They are associated with respiratory, neurological and systemic diseases. In swine, three coronaviruses have been described: transmissible gastroenteritis virus TGEV, porcine respiratory coronavirus (PRCV) and porcine epidemic diarrhea virus. TGEV and PRCV have 
been extensively studied and offer excellent animal models to understand mechanisms of coronavirus virulence and pathogenesis. PRCV, a coronavirus antigenically related to TGEV, was isolated in 1984 from pigs in Europe (Pensaert et al, 1986) and is believed to be a mutant of TGEV. TGEV causes gastroenteritis in neonatal pigs with a high mortality in pigs of 3 weeks of age or younger. PRCV causes respiratory disease in swine and is antigenically related to TGEV and is believed to be a mutant of TGEV. PRCV is ubiquitous in European swine herds and clinical TGE has become virtually nonexistent with concomitant increase in PRCV infection. PRCV was first isolated in 1988 in the United States (Hill et al., 1990). Now PRCV has been obtained from several swine herds in the U.S. (Paul et al., 1992, 1994; Vaughn et al., 1993; 1994, 1995; Wesley et al, 1991). We have also shown that PRCV is associated with pneumonia in swine. In this paper, some of the biological and nucleotide sequence differences observed among TGEV and PRCV isolates are presented. This data suggests that gene 3 of TGEV and PRCV may be important in viral virulence.

\section{TGEV AND PRCV HAVE DIFFERENT TISSUE TROPISM BUT ARE ANTIGENICALLY RELATED}

Pigs affected with TGE develop severe diarrhea within 24 hours after infection. Virus primarily replicates in villous epithelial cells of jejunum and ileum resulting in villous atrophy. PRCV primarily replicates in respiratory tract with limited to no replication in intestinal tract. PRCV has been shown to cause pneumonia after experimental inoculation of pigs intranasally (Halbur et al., 1993).

\section{TGEV AND PRCV ISOLATES VARY IN PATHOGENICITY}

TGEV isolates lose pathogenicity after cell culture passage. However, specific genetic changes have not been correlated with this loss in pathogenicity. Woods (1978) isolated a small plaque mutant (SP) of TGEV from virulent TGEV by passage of the virus in peripheral blood leukocytes derived fibroblast cell line. The SP mutant TGEV is nonpathogenic for neonatal pigs. This SP mutant virus has been experimentally shown to replicate in intestinal submucosa without significant replication in villous epithelial cells.

The extent of clinical disease caused by PRCV infections varies. The first isolates of PRCV were from clinically normal pigs and were thought to be non-pathogenic. Additional experimental studies have shown that some PRCV isolates can cause mild to moderate respiratory disease. The disease may be severe under field conditions especially in concurrent infections with other pathogens such as swine influenza virus and porcine reproductive and respiratory syndrome virus. We believe factors such as virus strain, virus concentration, and the age of the pigs impact virus pathogenicity.

The AR310 isolate was shown to produce moderate necrotizing and proliferative bronchointerstitial pneumonia in gnotobiotic pigs (Halbur et al., 1993). Similarly moderate bronchointerstitial pneumonia and clinical respiratory disease was observed in conventional 5-week-old SPF pigs inoculated experimentally with PRCV AR310 and another isolate IA-LEPP (Halbur et al., 1994 ). In contrast, PRCV IA-1894 caused only very mild bronchointerstitial pneumonia. These studies showed that there are differences in the pathogenicity of PRCV isolates. 


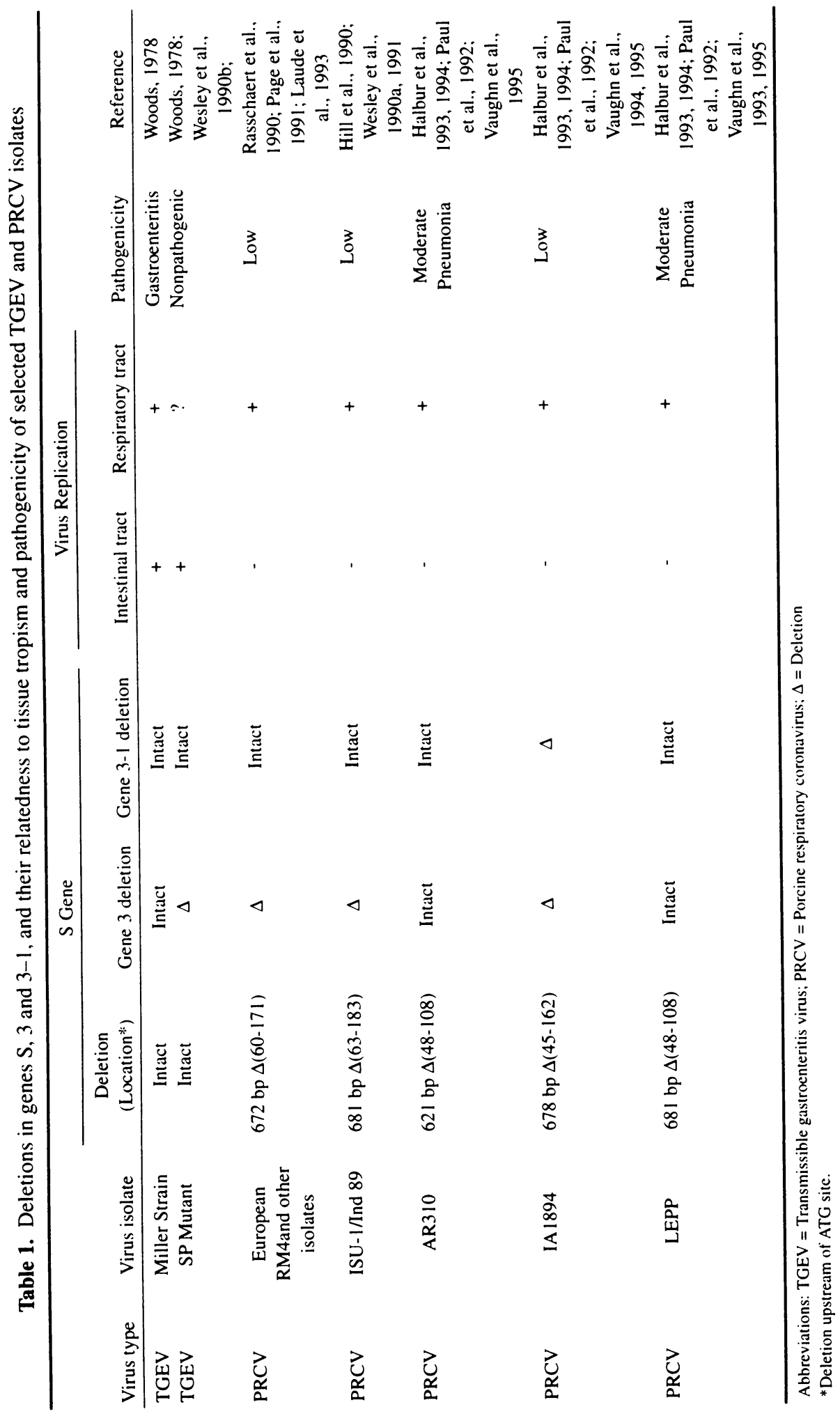




\section{PRCV IS A DELETION MUTANT OF TGEV}

All PRCV isolates characterized thus far have a deletion in the $5^{\prime}$-end of the $\mathrm{S}$ gene. The deletions in the $\mathrm{S}$ gene are 672 nucleotides in length for the European PRCV isolates and vary in size from $621-681$ nucleotides for the U.S. isolates (Table 1). The area deleted in all PRCV isolates characterized thus far includes amino acid residues of 92, 94, 218, and 219 which have been predicted by Sanchez et al. (1992) to be important in determining tissue tropism of PRCV and TGEV isolates. The location of the $\mathrm{S}$ gene deletion also varies in PRCV isolates as the deletion can start between nucleotides 45 and 63 and end between 108 and 183 upstream of the start site of the S gene of TGEV.

\section{GENE 3 IS ALTERED IN LOW VIRULENCE TGEV AND PRCV ISOLATES}

Genetic analysis has shown that genes 3 and/or 3-1 of some TGEV and PRCV isolates have deletions. There also appears to be correlation between the presence of gene 3 deletion and viral pathogenicity. The nonpathogenic SP mutant of TGEV virus has a large deletion in gene 3 (Wesley et al., 1990b). Similarly, gene 3 of the low virulence strains of PRCV have a deletion or substitution near the start site to make the ORF nonfunctional. Gene 3 was altered in PRCV strain IA-1894 of low pathogenicity whereas it was intact in PRCV isolates AR-310 and LEPP of high pathogenicity (Vaughn et al., 1995).

\section{CONCLUSIONS}

Evidence based on the comparison of virulence and nucleotide sequence suggests that there may be a genetic basis for differences in pathogenicity and tissue tropism of swine coronaviruses. The deletion in S gene may hamper the ability of virus to efficiently replicate in the intestinal tract. Gene 3 seems to play a role in virulence of TGEV and $\mathrm{PRCV}$ as virus isolates of low virulence have alterations in this gene. Additional studies are needed to further clarify the role of this gene in viral virulence.

\section{ACKNOWLEDGMENTS}

The authors thank Iowa Livestock Advisory Council for financial support of this study.

\section{REFERENCES}

Halbur, P. G., P.S. Paul, E.M. Vaughn, and J.J. Andrews. 1993. Experimental reproduction of pneumonia in gnotobiotic pigs with porcine respiratory coronavirus isolate AR310. J. Vet. Diagn. Invest. 5:184-188.

Halbur, P. G., P.S. Paul, and E.M. Vaughn. 1994. Virulent porcine respiratory coronavirus isolates exist in the United States. In Proc Inter Pig Vet Soc Congr, Bangkok, Thailand, p70.

Hill, H., J. Biwer, R. Wood, and R. Wesley. 1990. Porcine respiratory coronavirus isolated from two U.S. swine herds. Proc. Am. Assoc. Swine Practitioners. p333-335.

Laude, H., K. Van Reeth, and M. Pensaert. 1993. Porcine respiratory coronavirus: molecular features and virushost interactions. Vet. Res. 24:125-150. 
Page, K. W., K. L. Mawditt, and P. Britton. 1991. Sequence comparison of the 5' end of mRNA 3 from transmissible gastroenteritis virus and porcine respiratory coronavirus. J. Gen. Virol. 72:579-587.

Paul, P. S., E. M. Vaughn, and P. G. Halbur. 1992. Characterization and pathogenicity of a new porcine respiratory coronavirus strain AR310. Proc. Int. Pig Vet. Soc. Congr. 12:92.

Paul, P. S., P. G. Halbur, and E. M. Vaughn, 1994. Significance of Porcine Respiratory Coronavirus Infection. The Compendium of Continuing Education for Practicing Veterinarians, 16:1223-1245.

Pensaert, M., P. Callebaut, and J. Vergote. 1986. Isolation of a porcine respiratory, non-enteric coronavirus related to transmissible gastroenteritis. Vet. Quarterly. 8:257-261.

Rasschaert, D., M. Duarte, and H. Laude. 1990. Porcine respiratory coronavirus differs from transmissible gastroenteritis virus by a few genomic deletions. J. Gen. Virol. 71:2599-2607.

Sanchez, C. M., F. Gebauer, C. Sune, A. Mendez, J. Dopazo, and L. Enjuanes. 1992. Genetic evolution and tropism of transmissible gastroenteritis coronavirus. Virology. 190:92-105.

Vaughn, E.M., P.G. Halbur, and P.S. Paul. 1994. Three porcine respiratory coronavirus isolates with varying sizes of S gene deletions. J. Clin. Microbiol. 32:1809-1812.

Vaughn, E.M., P.G. Halbur, and P.S. Paul. 1995. Sequence Comparison of Porcine Respiratory Coronavirus Isolates Reveal Heterogeneity in S, 3, and 3-1 Genes. J. Virol. 69: 3176-3184.

Wesley, R. D., R. D. Woods, H. T. Hill, and J. D. Biwer. 1990a. Evidence for a porcine respiratory coronavirus, antigenically similar to transmissible gastroenteritis virus, in the United States. J. Vet. Diagn. Invest. 2:3i2-317.

Wesley, R. D., R. D. Woods, and A. K. Cheung. 1990b. Genetic basis for the pathogenesis of transmissible gastroenteritis virus. J. Virol. 64:4761-4766.

Wesley, R. D., R. D. Woods, and A. K. Cheung. 1991. Genetic analysis of porcine respiratory coronavirus, an attenuated variant of transmissible gastroenteritis virus. J. Virol. 65:3369-3373.

Woods, R.D. 1978. Small plaque variant transmissible gastroenteritis virus. J. Am. Vet. Med. Assoc. 173:643-647. 1978. 